\title{
La construction de l'identité des Setos (1920-1940)
}

The Building of Seto Identity (1920-1940)

Setode identiteedi konstrueerimine aastatel 1920-1940

\section{Antoine Chalvin}

\section{OpenEdition}

\section{Journals}

Édition électronique

URL : https://journals.openedition.org/efo/127

DOI : $10.4000 /$ efo. 127

ISSN : 2275-1947

\section{Éditeur}

INALCO

\section{Édition imprimée}

Date de publication : 1 janvier 2011

ISBN : 978-2-296-96641-3

ISSN : 0071-2051

\section{Référence électronique}

Antoine Chalvin, «La construction de l'identité des Setos (1920-1940) », Études finno-ougriennes [En ligne], 43 | 2011, mis en ligne le 28 mai 2014, consulté le 08 juillet 2021. URL : http:// journals.openedition.org/efo/127 ; DOI : https://doi.org/10.4000/efo.127

Ce document a été généré automatiquement le 8 juillet 2021

\section{(c) (†) 8}

Études finno-ougriennes est mis à disposition selon les termes de la Licence Creative Commons Attribution - Pas d'Utilisation Commerciale 4.0 International. 


\title{
La construction de l'identité des Setos (1920-1940)
}

\author{
The Building of Seto Identity (1920-1940) \\ Setode identiteedi konstrueerimine aastatel 1920-1940
}

Antoine Chalvin

1 Cet article retrace la politique d'annexion identitaire menée dans les années vingt et trente par l'État estonien à l'égard des Setos, un groupe de populations apparenté aux Estoniens. Ce processus peut être décrit comme une construction nationale volontariste pilotée de l'extérieur : les autorités estoniennes ont réussi à convaincre les Setos qu'ils étaient estoniens, alors qu'ils ne s'étaient pas encore préoccupés de savoir exactement ce qu'ils étaient (ils se définissaient surtout par leur religion orthodoxe, et non en termes nationaux). Parmi les méthodes mises en œuvre, outre la scolarisation massive en estonien, l'estonisation des noms propres et l'organisation de grands congrès populaires, les Estoniens se sont efforcés d'appliquer aux Setos des recettes issues de leur propre modèle de construction nationale, de reproduire avec eux de façon planifiée les évolutions qui avaient conduit, au siècle précédent, à la formation de leur conscience nationale : développement de la vie associative, développement de la presse, organisation de « fêtes du chant » et élaboration d'une épopée.

2 Les Setos, petite communauté ethnique installée à l'extrême sud-est de l'Estonie et audelà de la frontière actuelle avec la Russie, offrent un exemple intéressant d'un processus de construction identitaire lancé et piloté de l'extérieur, que l'on pourrait qualifier d'annexion identitaire. Son objectif était de conduire à la création d'une identité emboîtée, c'est-à-dire d'une identité ethnique étroite (l'identité seto) incluse dans une identité nationale plus large (l'identité estonienne). Ce processus s'est déroulé principalement pendant l'entre-deux-guerres, après l'accession de l'Estonie à l'indépendance. 

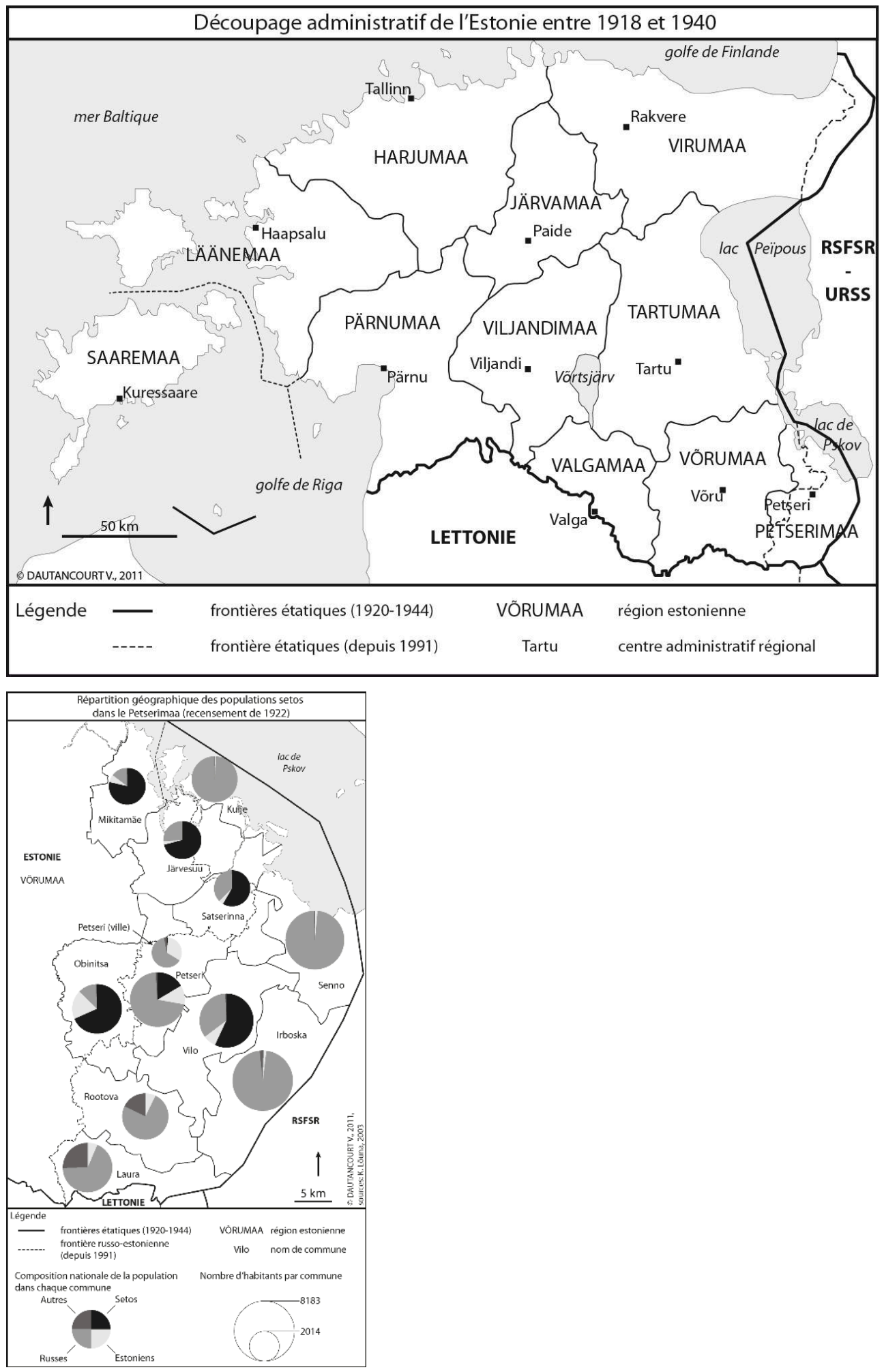

\section{L'origine des Setos}

Des théories divergentes ont été formulées au sujet de l'origine des Setos.

4 À la fin $\mathrm{du} \mathrm{XIX}{ }^{\mathrm{e}}$ siècle, les intellectuels russes de Pskov voyaient dans cette population le résultat de l'estonisation de vieux-croyants russes qui auraient fui la Russie pour 
échapper aux persécutions dont ils avaient été victimes à cause de leur foi (Hagu 1994 ; Hagu 1978, p. 616).

5 Plusieurs auteurs estoniens (Tampere 1956, Mägiste 1957) ont soutenu que les Setos étaient les descendants d'immigrants venus d'Estonie du sud. D'autres auteurs (Hurt 1904, Moora 1956) ont défendu quant à eux la thèse d'une origine autochtone.

6 Le débat entre ces deux dernières hypothèses n'est toujours pas tranché (l'origine russe est quant à elle tout à fait fantaisiste). On s'accorde toutefois sur le fait que la population seto aurait été alimentée de façon régulière, jusqu'au XIX siècle, par un flux migratoire en provenance d'Estonie du Sud, les nouveaux venus s'assimilant à la population locale. Ce processus d'assimilation aurait cessé de fonctionner au XIX siècle, car les nouveaux migrants avaient désormais une conscience nationale plus développée et la volonté plus forte qu'auparavant de conserver leur religion luthérienne (Lõuna 2003, p. 15).

\section{L'identité des Setos avant 1917}

7 La composante principale de l'identité des Setos avant 1917 était incontestablement la religion orthodoxe. Ils se percevaient avant tout comme des pratiquants de cette religion. Or, celle-ci était associée par eux à l'identité russe et à la langue russe : ils l'appelaient la « religion russe ». Nombre d'entre eux considéraient le russe (assimilé probablement au slavon) comme la langue de communication avec Dieu (Lõuna 2003, p.45). Cela permet de comprendre pourquoi ils se désignaient parfois comme des «Russes» (Jääts 1998, p.38), bien qu'ils eussent conscience de parler une langue différente. L'apparence vestimentaire des hommes (une chemise de couleur portée sur le pantalon) les rapprochait également des Russes des environs. Le costume et les bijoux des femmes, en revanche, étaient spécifiques.

8 Néanmoins, les Setos se mêlaient assez peu aux Russes et avaient à leur égard une attitude légèrement méprisante. On comptait par exemple très peu de mariages mixtes (Truusmann 2002, p. 181). Cela explique en partie l'absence de processus d'assimilation. Les Setos illettrés n'avaient pas véritablement de motivation pour s'assimiler aux paysans russes, qui avaient le même statut social qu'eux : la russification ne leur offrait en effet guère de possibilité de promotion sociale. Toutefois, les rares Setos qui accédaient à l'instruction avaient tendance à se russifier et à renier leurs origines. Ils se trouvaient dans une situation comparable à celle des Estoniens instruits qui, dans la première moitié du XIX ${ }^{\mathrm{e}}$ siècle, adoptaient la langue allemande.

9 Les Russes, de leur côté, ne reconnaissaient pas les Setos comme des Russes, mais les appelaient des « demi-croyants » (poluvertsy ou poluvernik), peut-être à cause de leurs coutumes païennes qui étaient encore bien vivantes au XIXe siècle (Truusmann 2002, p. 179). Les Setos rendaient notamment un culte à une divinité des récoltes et de la fertilité, Peko, culte qui s'est prolongé jusque dans les années trente et quarante du $\mathrm{xx}^{\mathrm{e}}$ siècle (Hõrn 1994). En outre, très souvent, ils ne connaissaient pas bien le russe, ni $a$ fortiori le slavon, langue de la liturgie orthodoxe. De ce fait, ils ne comprenaient sans doute pas grand-chose aux services religieux, ce qui explique peut-être aussi ce surnom (Jääts 1998, p. 31).

10 Les relations avec les Estoniens étaient également placées sous le signe de l'altérité. Les Estoniens percevaient les Setos comme des gens très différents et avaient à leur égard 
une attitude un peu condescendante. Leur langue était un sujet de moquerie. On se méfiait aussi beaucoup des marchands ambulants setos, qui avaient la réputation d'être des voleurs, et on refusait de les héberger chez soi. Les bagarres dans les tavernes entre Setos et Estoniens n'étaient pas rares (Kallas 1894, p. 183). Les Setos étaient également le sujet d'histoires drôles publiées par la presse estonienne, dans lesquelles ils apparaissaient généralement comme des ivrognes ou des imbéciles (Jääts 1998, p. 34-35).

11 Le nom Setu était d'ailleurs à l'origine un terme péjoratif utilisé par les Estoniens ${ }^{1}$. Les Setos eux-mêmes s'appelaient les "gens du pays" ou "gens de la campagne " (maarahvas), comme les Estoniens avant le milieu du XIx ${ }^{\mathrm{e}}$ siècle, ou utilisaient des désignations comportant un pronom de première personne du pluriel: "nous", "notre peuple », "notre langue ». Mais dès la fin du XIxe siècle, une partie d'entre eux commencèrent à utiliser le mot Seto pour se différencier des Russes et des Estoniens, selon un processus assez classique par lequel un peuple adopte pour se désigner luimême le nom que les autres lui donnent, c'est-à-dire convertit un exonyme en endonyme.

12 Il faut toutefois signaler que, dès la deuxième moitié du XIXe siècle, bon nombre de lettrés estoniens (linguistes ou folkloristes) avaient à l'égard des Setos une attitude très différente de celle de leurs compatriotes: loin de les mépriser, ils portaient au contraire un vif intérêt à leur langue et à leur folklore, qui faisaient d'eux, à leurs yeux, des Estoniens à part entière. Certains voyaient même dans le folklore seto un conservatoire du folklore estonien, où se seraient préservés à l'état pur et authentique des éléments qui auraient disparu ailleurs (cf. par exemple Hurt 1904, p. v).

Outre l'identité religieuse, étaient également importantes pour les Setos l'identité sociale (leur appartenance à la classe paysanne) et l'identité locale (l'appartenance à un village). L'identité linguistique n'avait en revanche qu'une importance secondaire (Jääts 1998, pp. 38-41). D'une part, la langue parlée par les Setos variait selon les villages, et ces différences locales semblent avoir été assez nettement perçues, ce qui, en l'absence d'une forme standard, empêchait la langue de fonctionner comme un facteur de cohésion identitaire. D'autre part, cette langue n'est pas spécifique aux Setos : au-delà des différences de détail, le critère de l'intercompréhension pourrait en effet rapprocher les Setos des Estoniens, notamment de ceux de la région de Võru, qui parlent un dialecte assez similaire, même si, comme on l'a vu, dans la pratique, la perception des différences était là aussi assez forte.

Cette population à la conscience nationale peu développée, située culturellement entre l'espace estonien et l'espace russe, va faire l'objet d'une véritable annexion identitaire par l'Estonie. Annoncé dès le xix siècle par l'attitude des lettrés nationalistes estoniens, le processus va véritablement commencer avec l'accession de l'Estonie à l'indépendance. La jeune république va mettre en quelque sorte en pratique la conception que les intellectuels estoniens se faisaient de l'origine et de l'identité des Setos, conception que l'on pourrait résumer en ces termes: les Setos sont des Estoniens, mais ils ne le savent pas, il faut donc les aider à en prendre conscience. 


\section{Le rattachement à l'Estonie et le redécoupage des communes} par les Setos. L'idée en avait été formulée dès 1864. Mais ce sont seulement les révolutions russes de 1917 qui la remirent à l'ordre du jour et permirent sa réalisation. Fin mars 1917, à la demande des Estoniens, le gouvernement provisoire russe accorda l'autonomie à l'Estonie, dont les frontières furent modifiées pour englober la plupart des régions peuplées par des Estoniens. La nouvelle entité administrative réunissait l'ancien gouvernorat d'Estonie (nord de l'Estonie actuelle) et le nord de l'ancien gouvernorat de Livonie (sud de l'Estonie actuelle). Mais le pays seto, qui appartenait au gouvernorat de Pskov, resta à l'écart de cette recomposition administrative. À l'été 1917, une "lettre des Estoniens de Petseri ou Setos", datée du $1^{\mathrm{er}}$ juillet et comportant 74 signatures, fut adressée à la Diète d'Estonie pour réclamer le rattachement de la province de Petseri au nouveau gouvernorat d'Estonie. Cette lettre appelait les Setos des «Estoniens » et exagérait nettement leur proportion dans la population totale des communes concernées $(80 \%$, alors qu'ils représentaient sans doute moins de $50 \%$ ) (Lõuna 2003, pp. 25, 150). Il semble que les auteurs de la lettre aient été en fait des Estoniens, et non des Setos (Jääts 1998, p. 42). La diète d'Estonie accueillit favorablement la demande, mais n'eut pas le temps de prendre des mesures concrètes, car la révolution d'Octobre empêcha la réalisation du projet.

Le 24 février 1918, l'Estonie proclama son indépendance "dans ses frontières historiques et ethnographiques ». La liste des provinces concernées figurant dans la déclaration d'indépendance ne mentionnait pas la province de Petseri, mais le texte précisait ensuite : « et les autres [provinces] où le peuple estonien est installé depuis longtemps et constitue la grande majorité.» Il précisait encore que la détermination exacte de la frontière avec la Russie et la Lettonie se ferait par référendum une fois la guerre terminée. Cette déclaration ne fut pas immédiatement suivie d'effets, puisque l'Estonie fut aussitôt occupée par l'Allemagne. L'indépendance effective fut conquise par les armes après le retrait des troupes allemandes : la jeune armée estonienne sortit victorieuse de la "guerre de Libération" contre l'armée rouge, puis contre la Landeswehr formée par les Germano-Baltes. Le 4 juin 1919, l'Assemblée constituante adopta une constitution provisoire en vertu de laquelle la province de Petseri (y compris des zones non peuplées par des Setos) faisait partie du territoire estonien. La frontière précise fut fixée par le traité de Tartu, signé entre l'Estonie et la Russie le 2 février $1920^{2}$. Le rattachement à l'Estonie de zones non peuplées par des Setos, qui apparaît en contradiction avec les principes de la déclaration d'indépendance (l'Estonie dans ses frontières ethnographiques), s'explique par les succès de l'armée estonienne et la volonté du gouvernement d'en profiter pour constituer une sorte de zone tampon avec la Russie.

Dans le territoire ainsi rattaché à l'Estonie, qui comptait près de 61000 habitants, les Russes étaient majoritaires (près de $64 \%$ de la population, d'après le recensement de 1922). Les Estoniens représentaient $7 \%$ et les Setos près de $25 \%$ (soit 15000 personnes) (Lõuna 2003, p. 33 ; Jääts 1998 , p. 43). Il faut noter que ce recensement, le premier organisé par la République d'Estonie, distinguait encore les Setos comme un groupe national différent des Estoniens. Ce sera le cas également en 1934. Mais dans les référendums ultérieurs (aussi bien à l'époque soviétique que dans l'Estonie 
indépendante), les Setos ne seront plus distingués des Estoniens, ce qui empêche d'avoir des données statistiques fiables sur leur évolution numérique et sur leur nombre actuel.

Compte tenu du fait que les Russes étaient largement majoritaires dans la province et dans les quatre communes initiales qui la constituaient, ce sont eux qui exerçaient le pouvoir au sein des instances communales et régionales. Cette situation était jugée néfaste pour les Setos, car elle risquait d'exercer sur eux une pression russificatrice (la langue de l'administration dans les communes était, de fait, le russe). Les autorités estoniennes estimaient au contraire nécessaire de favoriser le rapprochement des Setos avec les Estoniens et de leur permettre de prendre en main l'administration communale dans les zones où ils habitaient, afin de les soustraire à l'influence russe. La solution choisie pour y parvenir consista à procéder à un redécoupage administratif de la province, afin de former des communes relativement homogènes par leur composition ethnique, ou au moins de mettre les Setos en position majoritaire dans certaines d'entre elles. Le processus débuta en mars 1921 et aboutit en mai 1922, après des négociations parfois difficiles entre le pouvoir central et les autorités communales dominées par les Russes. Les quatre communes d'origine cédèrent la place à onze nouvelles entités (plus la ville de Petseri, qui n'était pas concernée par le redécoupage). Les Setos étaient majoritaires dans cinq d'entre elles (Järvesuu, Obinitsa, Mikitmäe, Satserinna, Vilo), où, avec les Estoniens, ils représentaient plus de $60 \%$ de la population. La proportion de Setos dans les autres communes était négligeable (moins de $1 \%$ ), sauf dans la commune de Petseri (16,6 \%) (Lõuna 2003, p. 34, tableau 1).

\section{Le développement de l'éducation}

L'école fut l'un des principaux vecteurs de l'estonisation des Setos.

Le niveau général d'instruction dans la région au début des années vingt était très bas : d'après le recensement de 1922 , seuls $44,3 \%$ de la population de plus de dix ans avait fréquenté l'école (contre 87,6\% pour l'ensemble de l'Estonie). Le taux d'analphabétisme était beaucoup plus élevé que dans le reste de l'Estonie : en 1922, 48,9\% des habitants du Petserimaa âgés de plus de dix ans ne savaient ni lire ni écrire (dans le reste de l'Estonie, ce taux était seulement de 3,4 \%) (Jääts 1998, p. 46). Il est intéressant de noter les différences entre les hommes et les femmes : celles-ci ne fréquentaient pas l'école, et la proportion d'illettrées parmi les femmes du Petserimaa s'élevait à $64 \%$ ). La fréquentation de l'école par les enfants était très faible. Dès l'année scolaire 1919/1920, le gouvernement rendit la scolarité obligatoire dans toute l'Estonie. Mais les Setos ne voyaient pas cette mesure d'un bon œil. Ils jugeaient que la scolarisation des filles n'était pas nécessaire : pour eux, une femme n'avait pas besoin de savoir lire et écrire. De façon générale, les enfants devaient participer aux travaux de la ferme, notamment en gardant les troupeaux, ce qui amenait souvent les parents à les garder à la maison ou à les retirer plus tôt de l'école au printemps et à les ramener plus tard à l'automne. Pour toutes ces raisons, la scolarité obligatoire fut longue à s'imposer dans les faits : en 1920/1921, seuls $55 \%$ des enfants de la province fréquentaient effectivement l'école. Le taux de scolarisation passera à $76 \%$ en 1922/1923, puis à $81 \%$ l'année suivante (Lõuna 2003, p. 52, graphique 4).

21 Les autorités mirent en œuvre une politique volontariste de création d'écoles. Alors que la province comptait 43 écoles primaires à l'automne 1919, leur nombre avait doublé 
(100) trois ans plus tard (Lõuna 2003, p. 49). Il baissera légèrement par la suite, du fait d'une meilleure organisation et de l'augmentation des capacités d'accueil des écoles. Pour permettre ce développement rapide du réseau scolaire, il fallut attirer des instituteurs. Pour cela, en septembre 1920, on décida de verser à tous les instituteurs travaillant dans la région un salaire supérieur de $50 \%$ au salaire en vigueur dans le reste de l'Estonie. Le nombre de maîtres d'école passa de 104 au printemps 1920 à 222 en 1931 (Lõuna 2003, p. 51). Les nouveaux instituteurs venaient évidemment des autres régions d'Estonie, et la majorité des écoles créées étaient de langue estonienne. Des écoles de langue russe furent également ouvertes, et elles continuèrent à représenter la majorité des établissements (58\% en 1931). Il existait même quelques écoles de langue lettone (trois en 1922/1923) (Lõuna 2003, p. 49).

La constitution estonienne de 1920 garantissait le droit des membres des minorités nationales à une éducation dans leur langue maternelle. Mais pour les Setos, cette disposition ne put s'appliquer. D'abord parce qu'il n'existait pas de langue écrite seto qui aurait pu servir de langue d'enseignement. Et ensuite parce que, de toute façon, les Setos étaient considérés par les autorités comme des Estoniens. On les obligea donc à fréquenter l'école estonienne, où l'enseignement était dispensé en estonien standard, fondé sur les dialectes estoniens du nord, très différents de la langue parlée par les Setos. Celle-ci est un dialecte de l'estonien du sud, lequel est aujourd'hui considéré par les linguistes comme une langue distincte de l'estonien du nord, et l'intercompréhension est effectivement très difficile. De fait, les enfants setos avaient de grandes difficultés à comprendre l'estonien standard (Lõuna 2003, p. 47). Certains Setos auraient préféré envoyer leurs enfants à l'école russe, au motif que le russe était davantage en accord avec leur religion (Raun 1991, p. 531). Au cours de l'année scolaire 1923/1924, les parents d'élèves d'une école primaire (à Saatse) écrivirent une lettre au ministre de l'Éducation pour réclamer le rétablissement du russe comme langue d'enseignement. Un détail amusant et révélateur est que, d'après leur lettre, ils semblaient croire que la langue d'enseignement alors utilisée dans leur école était l'allemand (Lõuna 2003, p. 49). Il peut s'agir d'une simple faute d'inattention, mais cela peut aussi indiquer qu'ils ne faisaient pas vraiment la différence entre l'allemand et l'estonien.

Conscients du problème, certains milieux intellectuels estoniens commencèrent à évoquer l'idée de créer une langue écrite seto et de publier des manuels scolaires dans cette langue, afin de permettre aux enfants d'apprendre à lire et de recevoir une instruction dans leur langue maternelle, au moins pendant leurs premières années d'école. Le projet fut discuté en 1921 à Tartu lors d'une réunion de la Société pour la langue maternelle (Emakeele Selts), mais il n'aboutit pas, parce qu'il se heurta à des résistances. La Société décida néanmoins de soutenir la réalisation d'un recueil de lectures en seto. Ce travail fut réalisé par un jeune Estonien de Räpina (ville d'Estonie du Sud, mais située en dehors du pays seto), Paulopriit Voolaine (1899-1985), étudiant à l'Université de Tartu. Son anthologie parut en deux volumes, publiés en 1922 et 1925. Pour pallier l'absence de littérature seto préexistante, l'auteur dut traduire en seto des textes estoniens, et écrire certains textes lui-même directement en seto (Hagu 1995, p.17). Le résultat, pour une première tentative, était apparemment très honorable. Mais ces recueils de textes ne furent jamais été utilisés dans les écoles. Le ministère de l'Éducation n'approuvait pas l'introduction du seto à l'école, car cela allait évidemment à l'encontre de l'objectif officiel d'estonisation (Lõuna 2003, p. 49). En outre, il semble que les Setos qui ont pris connaissance de ces manuels trouvaient que la langue n'était 
pas correcte, parce qu'elle présentait des différences avec celle qu'ils parlaient dans leur village. Il y avait donc chez eux une tendance à mettre en avant les différences dialectales, au lieu de les minimiser pour s'identifier à une langue écrite commune. C'est sans doute un signe d'une identité locale plus forte que l'identité nationale.

La scolarisation en estonien des enfants setos finit par porter ses fruits. En 1932/1933, dans son rapport d'inspection des écoles, le conseiller scolaire de la province signale, pour s'en féliciter, que parmi les élèves setos commence à apparaître " un sentiment d'appartenance à la grande famille estonienne ». Il précise également que les élèves eux-mêmes ne font plus de différence entre les Setos et les Estoniens (Lõuna 2003, p. 51).

Toutefois, cette intégration avait ses limites: les enfants setos parlaient estonien uniquement à l'école, ailleurs ils utilisaient toujours leur langue maternelle (ibid., p. 54).

Grâce à la scolarisation se forma une catégorie de Setos relativement instruits, une sorte d'intelligentsia seto. Certains Setos accédèrent même aux études supérieures. Près d'une quarantaine de Setos auraient ainsi étudié à l'université de Tartu entre 1918 et 1944 (Jääts 1998, p. 54). À partir des années trente, ces jeunes Setos instruits, porteurs de cette double identité (l'adhésion à une culture « savante » estonienne et la conscience de leur particularisme) aspiraient à prendre en main leur culture, à faire entendre leur voix propre, à la place des Estoniens qui parlaient et agissaient en leur nom. Ils étaient donc plutôt opposés à une estonisation complète. Il y eut en juillet 1933 et juillet 1934 des rencontres de jeunes Setos (60 participants la première année, 100 l'année suivante) qui débattirent de la situation des Setos dans la société estonienne et des mesures à prendre pour l'améliorer. Il y avait là un ferment qui aurait pu porter des fruits intéressants.

\section{L'estonisation des noms propres}

Dans les gouvernorats d'Estonie et de Livonie, les paysans avaient reçu des noms de famille au XIX ${ }^{e}$ siècle, après l'abolition du servage. Mais dans le gouvernorat de Pskov, cela n'avait pas été fait de façon systématique, et en 1920 la majorité des familles paysannes n'avaient pas de nom de famille. Les individus étaient désignés par leur prénom, complété par le nom du père, selon l'habitude russe, ou parfois par le nom de la ferme ou du village.

28 Le 29 juin 1921, un décret imposa aux habitants de la région qui en étaient encore dépourvus d'adopter un nom de famille, faute de quoi celui-ci leur serait attribué par le ministre de l'Intérieur. Une commission fut formée pour mettre en œuvre ce processus. Celui-ci se déroula en un temps assez bref, un peu plus de trois mois, du 19 septembre au 29 décembre 1921. Au total, ce sont environ 50000 personnes ( $80 \%$ de la population) qui reçurent un nom de famille ou modifièrent leur nom précédent. La majorité d'entre elles choisirent elles-mêmes leur nom (16 319 Setos et 27673 Russes). Pour les autres (2078 Setos et 3765 Russes), les noms furent attribués en tenant compte de l'appartenance nationale : les Russes se virent attribuer des noms russes (même si l'on dénombre une trentaine de cas où des noms estoniens furent attribués à des Russes), et les Setos se virent attribuer obligatoirement des noms estoniens, parfois même contre leur volonté. On en profita aussi pour estoniser des prénoms russes portés par les 
Setos : Ivan devint Ivo, Timofei devint Timmo, Stepan devint Teppo, etc. (ces prénoms, avec leur o en deuxième syllabe, sont typiques du sud de l'Estonie), ainsi que pour remplacer par des noms estoniens des noms de famille anciens à consonance russe (Lõuna 2003, p. 66).

Des toponymes russes furent peu à peu remplacés par des noms estoniens: les communes d'Obinitsa et d'Irboska furent rebaptisées respectivement Meremäe (1923) et Linnuse (1939). Les villages de Makarovo, Pokoldova et Gagarino furent rebaptisés Mäe, Tamme et Virve. Dans la ville de Petseri, la rue Uspenski devint la rue Tähe (« de l'étoile »), la rue Irboska devint la rue Aia (« du jardin »), etc.

En 1932, quelques conseillers municipaux de Petseri proposèrent même de donner un nom estonien à leur ville, car le nom actuel, bien qu'adapté, rappelait encore trop son étymologie russe (pechtchera $=$ caverne). Ils proposèrent d'appeler la ville Kalevilinn ("ville de Kalev »). Mais la proposition ne fut pas adoptée. Toutefois, en 1936, le parc boisé de Bogdanovka, en bordure de la ville, fut rebaptisé Kalevimägi («Colline de Kalev ») (Lõuna 2003, p. 92).

\section{L'estonisation de l'Église orthodoxe}

31 L'Église orthodoxe étant la principale source d'influence russe sur les Setos, il apparaissait nécessaire de promouvoir en son sein l'usage de la langue estonienne. Des tentatives furent faites, sur fond de relations complexes et assez conflictuelles entre l'État estonien et le clergé orthodoxe russe de la région. Le problème principal était le manque de prêtres estoniens. Au début des années vingt, le gouvernement nomma donc des prêtres estoniens payés par l'État, mais il dut finalement renoncer à cette politique sous la pression des partis de gauche, et la plupart des nouveaux prêtres quittèrent la région. Ensuite, une association locale financée par le ministère de l'Intérieur rémunéra un prêtre qui faisait des tournées dans les campagnes en célébrant des offices en estonien (Lõuna 2003, p. 45).

En 1933 fut créé le séminaire orthodoxe du Petserimaa. La moitié des séminaristes étaient estoniens et les autres devaient suivre des cours d'estonien.

En 1934, une loi réorganisa les paroisses sur une base nationale. De nombreux Setos appartenaient en effet à des paroisses mixtes comprenant également des Russes. Il y avait avant la réforme trois paroisses « estoniennes » (c'est-à-dire seto) et dix paroisses mixtes où les services religieux avaient généralement lieu en russe. Après la réforme, il y eut six paroisses estoniennes et huit paroisses russes (selon Lõuna 2003, p. 111 ; mais sept estoniennes et neuf russes selon Jääts 1998, p. 48), et plus aucune paroisse mixte. Il était également prévu qu'après le 15 juillet 1935, tous les offices religieux des paroisses estoniennes devaient avoir lieu en estonien.

\section{Les congrès des Setos}

À côté des mesures plus ou moins autoritaires imposées par le pouvoir central, comme la scolarisation des enfants setos en estonien et l'attribution de noms estoniens, il apparaissait nécessaire aussi de mobiliser les Setos eux-mêmes, de leur faire prendre conscience de leur identité estonienne et de les inciter à l'approfondir volontairement, et non plus sous la contrainte de la loi. L'un des principaux moyens employés pour cela 
fut l'organisation de "Congrès Setos ". Deux congrès eurent lieu dans l'entre-deuxguerres, en 1921 et en 1930. Ceux-ci étaient évidemment organisés à l'initiative d'Estoniens et consistaient essentiellement en des conférences prononcées par des Estoniens devant un auditoire de Setos relativement passif.

Le premier congrès (les 9 et 10 octobre 1921 à Petseri) rassembla environ deux cents participants, qui étaient des représentants des villages setos et des fonctionnaires communaux. L'objectif de ce congrès d'après l'un de ses organisateurs (Samuel Sommer) était de montrer aux Setos comment se libérer de la pression de la majorité russe. Il fallait pour cela, selon lui, rendre la vie économique et culturelle des Setos plus active, organiser des cours pour adultes afin de réduire l'illettrisme, propager le renoncement à l'alcool (les Setos buvaient beaucoup), fonder un journal et organiser un festival du chant. Un autre intervenant, le linguiste Villem Ernits, prononça une communication vigoureuse sur les dangers de l'alcool et proposa cinq mesures, parmi lesquelles la fermeture des tavernes et l'interdiction complète de la vente d'alcool. La conséquence fut que, dès le lendemain, les magasins d'alcool durent faire face à un afflux de clients sans précédent, car tout le monde voulait faire des réserves de peur que l'interdiction ne devienne effective (Lõuna 2003, pp. 67-68).

Les communications étaient prononcées en estonien standard, et les auditeurs setos avaient des difficultés à les comprendre.

Le deuxième congrès (le 30 mai 1930 à Petseri) avait toujours le même objectif : favoriser l'intégration des Setos dans la nation estonienne. Le nom officiel du congrès est d'ailleurs significatif: ce n'est plus "congrès seto ", mais "Congrès estonien ( $2^{\mathrm{e}}$ congrès seto) du Petserimaa». La relégation du mot seto entre parenthèses, au second plan derrière le mot estonien, montre bien la conception qui présidait à cette manifestation. Les organisateurs étaient des associations estoniennes de Petseri. Le nombre de participants (450) était beaucoup plus élevé que lors du premier congrès. L'événement était jugé important par les autorités, puisque le discours d'ouverture fut prononcé par le président du parlement estonien, Karl Einbund ${ }^{3}$. Celui-ci déplora la faible conscience nationale des Setos et déclara : «Le Seto doit devenir un Estonien, un citoyen à part entière. » Les autres interventions traitèrent notamment de la langue des Setos (Oskar Loorits), de la situation des écoles (K. Greenberg) et, cette fois encore, des ravages de la boisson (Villem Ernits). Les Setos restèrent là aussi dans un rôle assez passif. Aucun d'entre eux, semble-t-il, ne figurait parmi les intervenants, du moins à en juger par le compte rendu publié dans un journal local (Teine 1930).

Au total, les congrès ne semblent pas avoir eu d'effet direct sur les Setos. Ils ont surtout permis aux Estoniens de formuler des idées et des projets concernant l'estonisation de cette population. Au moins trois des moyens évoqués lors du premier congrès, et qui furent effectivement mis en œuvre, étaient directement inspirés par le réveil national estonien $\mathrm{du} \mathrm{XIX}^{\mathrm{e}}$ siècle : le développement de la presse, le développement de la vie associative et l'organisation d'une fête du chant choral. Les Estoniens s'efforçaient manifestement d'appliquer aux Setos leur propre modèle de construction nationale, de reproduire avec eux de façon planifiée les évolutions qui avaient conduit au siècle précédent à la formation de la conscience nationale estonienne. 


\section{La formation d'une presse locale}

$$
\text { d'une initiative du gouvernement estonien, mise en œuvre par l'intermédiaire de son }
$$
représentant extraordinaire dans la province (maaülem). L'ordre venait directement du ministre de l'Intérieur, Karl Einbund. Pour tenter d'assurer au journal des ressources suffisantes, on obligea les communes à s'y abonner et à y publier leurs annonces officielles. Le journal était publié par la chancellerie du représentant du gouvernement et financé par le ministère de l'Intérieur (Lõuna 2003, p. 69). Mais en novembre 1921, la fonction de représentant extraordinaire fut supprimée. Des Estoniens de Petseri tentèrent de poursuivre la publication en créant une société d'édition, mais faute de soutien financier de l'État, le projet ne put être réalisé et le journal cessa de paraitre en janvier 1922.

43 En 1923, une nouvelle tentative fut faite pour fonder un journal bilingue (Petseri Uudised), à l'initiative d'Estoniens de Petseri. Mais deux numéros seulement furent publiés.

C'est seulement en 1932 que commença à paraître le journal suivant, Vaba sõna (La libre parole), d'abord entièrement en estonien, puis très vite bilingue russe-estonien. Une partie de la rédaction fit scission et fonda la même année un nouveau journal reprenant le titre Petseri Uudised (Les nouvelles de Petseri), dirigé et financé par le maire de la ville, August Ruusmaa (Lõuna 2003, p. 89). La concurrence fut fatale à Vaba sõna, qui disparut en septembre. Petseri Uudised continua de paraître jusqu'à la fin de l'année 1937.

45 Ces journaux en estonien défendaient des points de vue nationalistes, et manifestaient une certaine hostilité aux Russes de la région. Leur influence sur l'identité des Setos n'a pas été étudiée précisément, mais il ne semble pas qu'elle ait été très importante, car les Setos n'étaient probablement pas de grands lecteurs de journaux (même dans les années trente, le taux d'illettrisme restait supérieur à la moyenne estonienne). En outre, ces journaux étaient rédigés par des Estoniens, et non par les Setos eux-mêmes. Il est donc probable que leur contenu ne reflétait pas les préoccupations et les centres d'intérêt de ces derniers. 


\section{Le développement de la vie associative et culturelle}

C'est en fondant des associations de toutes sortes (chorales, troupes de théâtre, associations sportives, unions professionnelles, coopératives, sociétés de crédit mutuel, etc.) que les Estoniens, dans la seconde moitié du XIX ${ }^{e}$ siècle, avaient développé leurs capacités d'organisation et leur cohésion nationale. Une évolution de ce type commença dans la région du Petserimaa dans les années vingt, aussi bien chez les Russes que chez les Setos, dans ce dernier cas avec le soutien des Estoniens. Ces associations étaient pour la plupart organisées sur une base nationale : on trouvait d'un côté des associations "russes", de l'autre des associations "estoniennes" qui regroupaient les Estoniens et étaient ouvertes également aux Setos. Le gouvernement accordait des financements à ces associations, sans oublier les Russes, mais en privilégiant tout de même les associations estoniennes (Lõuna 2003, p. 97). Le gouvernement créa surtout, en 1924, une association rattachée au ministère de l'Intérieur et destinée à mettre en œuvre sur le terrain la politique d'estonisation et de développement économique et éducatif des Setos, l'Association des régions frontières (Piirimaade Selts). Celle-ci comptait comme membres d'honneur le ministre de l'Intérieur, Karl Einbund, et le Premier ministre, Konstantin Päts. L'association eut une activité importante et encouragea notamment la création d'associations économiques locales dans les communes. Dans les années vingt, les Setos participaient surtout à ces associations économiques et de crédit, beaucoup moins aux associations culturelles, et encore moins aux associations de tempérance !

La principale activité culturelle spécifique aux Setos était le chant choral, principalement les chorales de femmes. Le chant traditionnel seto était en effet très particulier, et nettement distinct du chant traditionnel estonien, notamment par son caractère polyphonique. Les chorales seto devinrent très actives et commencèrent même à se produire dans le reste de l'Estonie. Plusieurs chanteuses au répertoire particulièrement riche suscitaient un vif intérêt de la part des folkloristes. Conformément aux projets présentés lors du premier congrès seto, des activistes estoniens (Samuel Sommer et Villem Ernits) organisèrent le 15 octobre 1922 une fête des chanteurs populaires setos, et le 22 juin 1924 la première fête générale du chant choral seto (appelée à l'époque "Deuxième fête du chant du Petserimaa", cf. Petserimaa 1924). Là aussi, on perçoit nettement l'influence du modèle estonien de construction nationale : la première fête panestonienne du chant choral, en 1869, avait en effet joué un rôle décisif dans la formation de la conscience nationale.

La première de ces fêtes fut financée par le ministère de l'Intérieur, dont la dotation permit de rémunérer très convenablement les chanteurs. La deuxième, probablement financée aussi par des fonds publics, rassembla des chorales seto de toute la province, en tout trois cents chanteurs qui se produisirent devant sept cents auditeurs, parmi lesquels de hautes personnalités: le ministre de l'Intérieur et son prédécesseur, le ministre de l'Éducation et l'homme politique Jaan Tõnisson (Lõuna 2003, p. 97). Cela montre l'importance que les autorités estoniennes attachaient à cet événement, qui devait selon elles contribuer au réveil national des Setos et les aider à prendre conscience de leur estonitude.

Je manque d'éléments pour évaluer précisément l'influence directe de ces manifestations sur l'identité des Setos, mais il est probable que c'est pendant ces années-là, grâce aux fêtes du chant, grâce aussi à l'intérêt des folkloristes, que les Setos 
ont pris conscience que le chant choral était une composante essentielle de leur identité et ont commencé à survaloriser cette activité.

\section{Le projet d'épopée} avait contribué de façon essentielle au processus de construction nationale, Paulopriit Voolaine, dont il a déjà été question plus haut, pensait qu'une épopée pourrait contribuer à éveiller la conscience nationale des Setos. Dans le deuxième volume de son recueil de lectures en seto (1925), il avait inclus des poèmes lyrico-épiques dont il était l'auteur, et dont il voulait probablement faire la base d'une épopée. Ces textes étaient en effet publiés sous le titre : «Fragments du récit "Le Roi Seto" ». À cette époque, il envisageait d'écrire lui-même cette épopée. Mais le résultat était assez éloigné de la poésie populaire authentique : le style de l'auteur était trop sentimental et romantique. Apparemment, Voolaine s'en rendit compte, car il renonça à son projet initial (Hagu 1995, p. 18-21).

51 En 1926 ou 1927, il rencontra Anne Vabarna (1877-1964), qui était incontestablement la plus grande chanteuse populaire seto, "découverte» en 1923 par le folkloriste finlandais Armas Otto Väisänen. On aurait recueilli de sa bouche environ 150000 vers, ce qui la place au premier rang des chanteurs populaires de toute l'aire fennique (à titre de comparaison, celle qui vient en seconde position, l'Ingrienne Larin Paraske, a livré aux folkloristes 32000 vers, soit presque cinq fois moins). En plus de sa connaissance d'un grand nombre de chants traditionnels, Anne Vabarna pratiquait aussi avec une grande aisance l'improvisation: elle inventait au fur et à mesure les paroles de ses chansons, sur des mélodies traditionnelles (Hagu 1995, pp. 30-31).

Cest probablement à l'époque de sa rencontre avec Anne Vabarna que Voolaine imagina la méthode idéale pour obtenir une épopée populaire seto authentique : en février 1927, il envoya à la chanteuse une lettre dans laquelle il lui indiquait les grandes lignes d'un récit qu'il avait imaginé et lui demandait d'improviser des chants en suivant ce canevas narratif. Il avait choisi de nommer le personnage principal Peko ou Pekolanõ. Il s'agissait pour lui d'un être humain doté d'une grande force et couronné par Jésus roi des Seto. Mais le nom est aussi, comme nous l'avons vu, celui du dieu de la fertilité et des récoltes dans la mythologie des Seto. Le personnage de Peko conçu par Voolaine possède certaines caractéristiques empruntées à la divinité païenne (il fait pousser les céréales grâce à sa massue magique).

53 Anne Vabarna, après avoir reçu cette lettre, se mit au travail presque aussitôt. Comme elle était illettrée, c'est son fils, âgé de dix-neuf ans, qui écrivit le texte sous sa dictée. L'épopée (environ 8000 vers) fut achevée au mois de mai de la même année (Hagu 1995, p. 36). Malheureusement, elle ne put être publiée et resta longtemps à l'état de manuscrit. Elle ne put donc pas jouer à l'époque le rôle que Voolaine aurait souhaité. C'est seulement, en 1995 que l'épopée fut enfin publiée (Peko 1995) et trouva sa place dans la culture des Setos.

Les circonstances particulières de sa composition donnent à Peko des traits assez originaux par comparaison avec les épopées élaborées par des lettrés à partir de matériaux folkloriques. Alors que le canevas proposé par Voolaine aurait permis de faire du personnage principal, Peko, un héros épique romantique, un chef de peuple porteur de l'identité nationale, Anne Vabarna ne s'engagea pas dans cette voie, qui est 
étrangère à son univers et aux chants traditionnels qu'elle connaît. Elle s'attacha surtout à décrire la vie quotidienne de Peko, ses travaux agricoles, les coutumes accompagnant la naissance, le mariage, la mort, les sentiments des personnages féminins, etc. La dimension épique proposée par le canevas de Voolaine est donc peu développée. Peko n'est pas une véritable épopée héroïque, même si elle comporte un héros et quelques batailles. Il s'agit plutôt de ce qu'on pourrait appeler une épopée paysanne du quotidien.

\section{L'identité seto à la veille de la Seconde Guerre mondiale}

La plupart des grandes initiatives qui ont contribué à la construction de l'identité seto, en tant qu'identité emboîtée au sein de l'identité estonienne, ont été lancées par des Estoniens. Les Setos eux-mêmes, dépourvus de conscience nationale forte, se sont laissés faire sans trop résister et ont adopté peu à peu ce modèle identitaire qu'on leur proposait.

Ce processus a eu pour résultat une évolution incontestable de l'identité seto, mais apparemment de façon très différente selon les générations. L'estonisation était évidemment beaucoup plus poussée chez les jeunes qui avaient fréquenté l'école estonienne et, plus particulièrement chez les garçons, qui avaient fait leur service militaire dans l'armée estonienne. On peut dire que l'identité seto était alors une identité en transition. Cela explique que les évaluations qui en sont fournies varient selon les auteurs. Selon un voyageur de la fin des années trente, Wladimir Bucholtz, les jeunes Setos avaient abandonné les coutumes traditionnelles propres à leur culture, et bon nombre d'entre eux se considéraient comme des Estoniens (cité par Lõuna 2003, p. 117). Selon l'ethnologue Indrek Jääts (Jääts 1998, pp. 57-58), les Setos, à la fin des années trente, avaient encore conscience de leur singularité, ils ne s'étaient estonisés que partiellement, et ce sera seulement plus tard, à l'époque soviétique, que le processus engagé entre les deux guerres trouvera son aboutissement. Selon lui, il n'y a pas eu entre les deux guerres de remplacement d'une identité par une autre, parce que les trois conditions nécessaires à un tel changement n'étaient pas remplies. Jääts s'appuie sur la théorie de Samuel Huntington, pour qui un changement d'identité civilisationnelle n'est possible que si trois conditions sont remplies : 1) l'élite du groupe doit soutenir le changement, 2) la majorité doit l'accepter ; 3) les forces dominantes de la civilisation d'accueil doivent accepter les nouveaux venus. Or, pour Jääts, 1) la petite élite seto était opposée à l'estonisation et souhaitait conserver sa singularité, 2) la majorité des Setos n'ont suivi le mouvement qu'à contrecœur et passivement, 3) les Estoniens n'étaient pas disposés à accepter les Setos parmi eux et conservaient à leur égard un sentiment de supériorité.

57 Mais un autre auteur, l'historien Kalle Lõuna, conteste ces trois affirmations de Jääts. Pour lui, les Estoniens étaient globalement bienveillants à l'égard des Setos et souhaitaient les accueillir dans « la grande famille estonienne ». La majorité des Setos, malgré une certaine passivité et des oppositions ponctuelles, a suivi le mouvement. Et enfin, dans les années trente, l'élite intellectuelle seto n'était pas opposée à l'estonisation, mais plutôt à ceux qui la mettaient en œuvre, qui étaient perçus comme des mercenaires culturels au service du gouvernement. Elle voulait simplement prendre en main le destin culturel des Setos, mais sans remettre en question leur 
rapprochement avec les Estoniens. Ce jugement est confirmé par le slogan adopté par les jeunes intellectuels Setos qui avaient fondé en 1930 une "Union éducative du Petserimaa ", qui rassemblait une quinzaine d'associations culturelles. Parodiant le slogan célèbre des jeunes intellectuels estoniens du début du siècle («Restons estoniens, mais devenons aussi européens!»), ils avaient résumé leurs aspirations identitaires en déclarant : « Devenons estoniens, devenons européens, mais restons des Setos!» (Lõuna 2003, p. 100). Devenir estoniens tout en restant des Setos, cela résume parfaitement, il me semble, la mutation identitaire subie par ce groupe de population pendant cette période.

\section{L'évolution ultérieure : de 1940 à nos jours}

Bien que le cadre chronologique de la présente étude s'arrête en 1940, il n'est pas inintéressant de montrer les prolongements de la politique d'annexion identitaire que nous venons de décrire, en retraçant brièvement l'évolution de l'identité seto de la Seconde guerre mondiale à nos jours.

En 1945, alors que l'Estonie est devenue une république soviétique, la majeure partie du Petserimaa est rattachée administrativement à la République socialiste fédérative de Russie. De nombreux Setos se retrouvent donc du côté russe. Mais cela ne modifie pas fondamentalement leur vie quotidienne, puisqu'il ne s'agit encore à l'époque que d'une frontière administrative, qu'ils peuvent franchir librement.

Pendant la période soviétique, le processus d'estonisation s'accélère et s'intensifie, au risque de remettre en question l'identité seto. Cela est dû à une série de facteurs.

61 En premier lieu, les instances officielles cessent de valoriser, et même de reconnaître la particularité des Setos. Ceux-ci cessent de constituer une nationalité reconnue. Lors des recensements, ils ne peuvent plus se déclarer Setos. Les seules catégories nationales qui leur sont proposées sont « Estonien » et « Russe ».

62 En second lieu, la religion perd de son importance, notamment auprès des jeunes. Or, c'était, comme on l'a dit, un vecteur identitaire essentiel. Les Setos qui cessent de se reconnaître orthodoxes perdent une caractéristique culturelle qui les différenciait des Estoniens.

63 L'estonisation complète de nombreux Setos a également été favorisée par l'exode rural, qui les conduit à s'installer dans des villes ou dans d'autres régions rurales d'Estonie. C'est particulièrement le cas de ceux d'entre eux qui ont fait des études.

Parallèlement à l'estonisation croissante d'une partie des Setos, se déroule également un processus de russification, qui affecte principalement ceux qui habitent dans la partie rattachée à la Russie et choisissent de ne pas déménager de l'autre côté de la frontière administrative.

65 Le rétablissement de l'indépendance de l'Estonie, le 20 août 1991, pose de graves problèmes aux Setos, du fait de la transformation de la frontière administrative en frontière d'État. En s'appuyant sur le traité de Tartu de 1920, des activistes réclament le rattachement à l'Estonie des régions annexées par la Russie. Mais le gouvernement estonien, pour tenter d'améliorer ses relations avec la Russie, finit par renoncer officiellement aux frontières fixées par le traité de Tartu.

Dès la fin des années quatre-vingt, on assiste à la renaissance du particularisme seto et d'une vie culturelle locale très animée. Cette évolution est liée à la naissance d'une 
identité régionale plus vaste, qui englobe également la région du Võrumaa, dont les habitants parlent une langue très proche. Une nouvelle langue écrite voit le jour, qu'on baptise du nom de võro-seto. Des associations sont créées pour promouvoir la culture et défendre les intérêts des Setos. Plusieurs congrès setos sont organisés (1993, 1996, 1999), qui prolongent la tradition inaugurée dans l'entre-deux-guerres, mais cette fois ce sont les Setos eux-mêmes qui les organisent. Un musée de la culture populaire seto est créé à Värska. Les chorales retrouvent une intense activité. Chaque année depuis 1994, au mois d'août, ont lieu les "Journées du royaume seto", une grande fête populaire au cours de laquelle est élu l'ülemsootska, c'est-à-dire le représentant sur terre du roi des Setos, Peko. Les Setos se sont également dotés de leur drapeau, de leur hymne. L'épopée seto a enfin été publiée. Il existe des émissions de radio en seto.

On peut dire qu'après le rétablissement de l'indépendance, le processus de construction identitaire seto a repris au point où il s'était arrêté en 1940 : le modèle est toujours celui de la double identité (la plupart des Setos ne remettent pas en cause leur appartenance au peuple estonien, même si certains activistes considèrent les Setos comme un peuple distinct), et la volonté de prise en main du processus par les Setos eux-mêmes, qui avait commencé à s'exprimer dans les années trente, s'est concrétisée. Le principal héritage de la période soviétique est la forte diminution du nombre de personnes se définissant comme des Setos. Ce nombre reste difficile à déterminer avec précision. Mais certaines sources l'estiment entre 10000 et 13000 , dont 3000 à 4000 dans le Setomaa en Estonie (EstBLUL), tandis que le recensement russe de 2002 en dénombre environ 200 dans la Fédération de Russie (SFSP).

\section{BIBLIOGRAPHIE}

EstBLUL = Estonian Bureau for Lesser-Used Languages, fiche sur la langue seto.

HAGU Paul, 1978, « Setode etnogenees agraartavandi valgusel », Keel ja kirjandus, ${ }^{\circ}$ 10, pp. 616-623.

HAGU Paul, 1994, «Setokaisten identiteetin ongelmat », Viro-Tietoutta, n 7, Helsinki : Tuglas Seura.

Hagu Paul, 1995, "Saateks », in Peko : Seto rahvuseepos, Setokaiseepos, The Seto epic, Snellmaninstituutin julkaisuja, A 18, Kuopio : Snellman-instituutti, pp. 15-39.

HõRn Aare, 1994, « Setokaisten ortodoksisuus ennen ja nyt », Viro-Tietoutta, n 6, Helsinki : Tuglas Seura.

HURT Jakob, 1904, « Eeskõne », in Setokeste laulud : Pihkva-eestlaste vanad rahvalaulud, ühes Räpinä ja Vastseliina lauludega, I, Helsinki : Soome Kirjanduse Selts, pp. v-xii.

JÄÄTS Indrek, 1998, Setode etniline identiteet, Studia ethnologica tartuensia, 1, Tartu : Tartu Ülikooli etnoloogia õppetool, $174 \mathrm{p}$.

KALLAS Oskar, 1894, « Üht ja teist setodest », Eesti Üliõpilaste Seltsi album, II, pp. 174-188. 
KeEM Hella, 1997, Võru keel, Tallinn : Emakeele Selts.

LõunA Kalle, 2003, Petserimaa : Petserimaa integreerimine Eesti Vabariiki, 1920-1940, Tallinn : Eesti Entsüklopeediakirjastus, $174 \mathrm{p}$.

MÄGISTE Julius, 1957, « Petserimaast, selle uurimisest ja setode päritolust », in Meie maa : Eesti sõnas ja pildis, IV, Lõuna-Eesti, Lund : Eesti Kirjanike Kooperatiiv, pp. 165-173.

MINAUDIER Jean-Pierre, 2007, Histoire de l'Estonie et de la nation estonienne, Paris : ADÉFO-

L'Harmattan.

Moora Harri, 1956, « Eesti rahva ja naaberrahvaste kujunemisest arheoloogia andmeil », in Eesti rahva etnilisest ajaloost, Tallinn : Eesti Riiklik Kirjastus, pp. 41-119.

Peko 1995= Peko: Seto rahvuseepos, Setokaiseepos, The Seto epic, Snellman-instituutin julkaisuja, A 18, Kuopio: Snellman-instituutti, 1995, pp. 15-39.

Petserimaa 1924= «Petserimaa 2. laulupidu », Võru teataja, 28 juin 1924, p. 2.

Raun Toivo U., 1991, “The Petseri region of the Republic of Estonia”, Jahrbücher für Geschichte Osteuropas (Stuttgart), 39, H. 4, pp. 514-532.

SFSP = Service fédéral des statistiques publiques (Russie), tableau de la composition nationale de la Fédération de Russie.

TAMPERE Herbert, 1956, « Mõningaid eestlaste etnilise ajaloo küsimusi suulise rahvaloomingu valgusel », in Eesti rahva etnilisest ajaloost, Tallinn : Eesti Riiklik Kirjastus, pp. 255-277.

Teine $1930=$ « Teine Seto kongress Petseris », Võru teataja, nº 61, 3 juin 1930, p. 2.

TRUUSMANN Jüri (Georg), 2002, « Petserimaa setod », Mäetagused, n 19, pp. 176-190.

\section{NOTES}

1. Selon une explication répandue (cf. Keem 1997, p. 20), le mot viendrait d'une déformation du partitif du pronom démonstratif (seta/setä). Il aurait été formé dans la première moitié du $\mathrm{XVIII}^{\mathrm{e}}$ siècle. Les habitants de la région de Petseri (en russe Petchory) achetaient à cette époque des pots et autres récipients en terre cuite à une fabrique de Petseri, et allaient les échanger contre des chiffons dans d'autres régions d'Estonie. Après quoi ils vendaient les chiffons à la fabrique de papier de Räpina. Lors de ces tournées, les contacts avec les autres Estoniens se heurtaient parfois à des difficultés linguistiques, et pour préciser les souhaits des clients, les vendeurs de pots devaient demander : « Kas taht setä vai setä ? (Est-ce que tu veux ça ou ça ?). Le mot setä, qui revenait sans cesse dans leur discours, a été modifié par contamination avec une autre particularité de leur langue (la présence de la voyelle o ailleurs que dans la première syllabe), pour donner le mot seto, par lequel les Estoniens ont commencé à les désigner.

2. Pour un aperçu historique plus détaillé de cette période, voir par exemple Minaudier 2007, pp. 239-253.

3. Il estonisera son nom en 1935 pour devenir Kaarel Eenpalu. 


\section{RÉSUMÉS}

Cet article retrace la politique d'annexion identitaire menée dans les années vingt et trente par l'État estonien à l'égard des Setos, un groupe de population apparenté aux Estoniens. Ce processus peut être décrit comme une construction nationale volontariste pilotée de l'extérieur : les autorités estoniennes ont réussi à convaincre les Setos qu'ils étaient estoniens, alors qu'ils ne s'étaient pas encore préoccupés de savoir exactement ce qu'ils étaient (ils se définissaient surtout par leur religion orthodoxe, et non en termes nationaux). Parmi les méthodes mises en œuvre, outre la scolarisation massive en estonien, l'estonisation des noms propres et l'organisation de grands congrès populaires, les Estoniens se sont efforcés d'appliquer aux Setos des recettes issues de leur propre modèle de construction nationale, de reproduire avec eux de façon planifiée les évolutions qui avaient conduit, au siècle précédent, à la formation de leur conscience nationale : développement de la vie associative, développement de la presse, organisation de "fêtes du chant » et élaboration d'une épopée.

This article analyses the process of identity building through which the young Estonian Republic, during the interwar period, tried to integrate the Setos, a small ethnic group related to Estonians and living in the far south-east of Estonia, into the Estonian ethnos. This process can be described as a voluntarist national construction directed from the outside: the Estonian authorities succeeded in convincing the Setos that they were Estonians, although they had not yet begun to perceive themselves as a nation (their self-identity was based mainly on their belonging to the Orthodox Church and not on a national consciousness). Among the methods applied, apart from Estonian language education for children, were the estonianisation of first names and family names and the organising of large popular congresses. Estonians tried to apply to the Setos recipes that were inspired by their own nation building process, i.e. to reproduce with the Seto in a planned manner the evolutions which had led in the previous century to the formation of their own national consciousness: development of associations, development of the press, and organisation of song festivals and elaboration of an epic.

Artiklis analüüsitakse Eesti riigi poliitikat setode identiteedi konstrueerimisel kahe sõja vahelisel perioodil. Seda protsessi võib kirjeldada väljastpoolt juhitud rahvusliku ärkamisena: Eesti võimud suutsid setosid veenda, et nood on eestlased, kuigi setod ei tajunud end veel rahvusena (nende enesemääratlus põhines eelkõige õigeusul, mitte rahvuslikul kuuluvusel). Lisaks sellele, et lastele anti eestikeelset kooliharidust, eestipärastati setode isikunimesid ning korraldati suuri rahvuslikke konverentse, püüdsid eestlased rakendada setode juures niisuguseid meetodeid, mis lähtusid eesti rahvusidentiteedi konstrueerimise mudelist : sooviti teadlikult uuesti esile kutsuda arenguid, tänu millele oli eelmisel sajandil sündinud eesti rahvuslik teadvus. Nende meetodite hulgas olid näiteks seltsielu elavdamine, ajakirjanduse arendamine, laulupidude korraldamine ning eepose loomine. 
INDEX

Index chronologique : XXe siècle

nomsmotscles Germano-Baltes, Seto

Keywords : identity, identity construction, ethnic awakening, Orthodoxy, Estonia, Seto country, Russia, Twentieth century, interwar years, history

Thèmes : histoire

motscleset Identiteet, identiteedi construeerimine, rahvusärkamine, õigeusk, Eesti, Setomaa, Venemaa, 20. Sajand, eesti aeg, kahesõjavaheline aeg, ajalugu

Mots-clés : identité, construction identitaire, éveil national, orthodoxie

Index géographique : Estonie, Pays seto, Fédération de Russie, Allemagne, Järvesuu, Mikitmäe, Obinitsa, Petseri, Pskov (oblast'), Räpina, Satserinna, Tartu, Värska, Vilo, Võru, Võrumaa 